\title{
Acute Kidney Injury and Special Considerations during Renal Replacement Therapy in Children with Coronavirus Disease-19: Perspective from the Critical Care Nephrology Section of the European Society of Paediatric and Neonatal Intensive Care
}

\author{
Akash Deep $^{\text {a }}$ Mehak Bansal $^{\text {b }}$ Zaccaria Ricci $^{c}$ \\ aPaediatric Intensive Care Unit, King's College Hospital NHS Foundation Trust, London, UK; bPediatric Intensive Care \\ Unit, SPS Hospitals, Ludhiana, India; 'Pediatric Cardiac Intensive Care Unit, Bambino Gesù Children's Hospital, IRCCS, \\ Rome, Italy
}

\section{Keywords}

Acute kidney injury · Pediatrics · Pandemic · Renal

replacement therapy $\cdot$ Coronavirus

\begin{abstract}
Children seem to be less severely affected by severe acute respiratory syndrome coronavirus-2 (SARS-CoV-2) as compared to adults. Little is known about the prevalence and pathogenesis of acute kidney injury (AKI) in children affected by SARS-CoV-2. Dehydration seems to be the most common trigger factor, and meticulous attention to fluid status is imperative. The principles of initiation, prescription, and complications related to renal replacement therapy are the same for coronavirus disease (COVID) patients as for nonCOVID patients. Continuous renal replacement therapy (CRRT) remains the most common modality of treatment. When to initiate and what modality to use are dependent on the available resources. Though children are less often and less severely affected, diversion of all hospital resources to manage the adult surge might lead to limited CRRT re-
\end{abstract}

sources. We describe how these shortages might be mitigated. Where machines are limited, one CRRT machine can be used for multiple patients, providing a limited number of hours of CRRT per day. In this case, increased exchange rates can be used to compensate for the decreased duration of CRRT. If consumables are limited, lower doses of CRRT (15$20 \mathrm{~mL} / \mathrm{kg} / \mathrm{h}$ ) for $24 \mathrm{~h}$ may be feasible. Hypercoagulability leading to frequent filter clotting is an important issue in these children. Increased doses of unfractionated heparin, combination of heparin and regional citrate anticoagulation, or combination of prostacyclin and heparin might be used. If infusion pumps to deliver anticoagulants are limited, the administration of low-molecular-weight heparin might be considered. Alternatively in children, acute peritoneal dialysis can successfully control both fluid and metabolic disturbances. Intermittent hemodialysis can also be used in patients who are hemodynamically stable. The keys to successfully managing pediatric AKI in a pandemic are flexible use of resources, good understanding of dialysis techniques, and teamwork.

(c) 2020 S. Karger AG, Basel

$\begin{aligned} & \text { karger@karger.com } \\ & \text { www.karger.com/bpu }\end{aligned}$
Karger ${ }^{\prime /}$




\section{Introduction}

Novel coronavirus disease (COVID-19) caused by severe acute respiratory syndrome coronavirus-2 (SARSCoV-2), first emerged in Wuhan, China, in December $2019[1,2]$. Shortly afterward, it was declared a global pandemic by the World Health Organization (WHO). As this virus has traveled across the world, it has overwhelmed many health care systems. As of May 3, 2020, $3,349,786$ cases of COVID-19 have been reported with 238,628 deaths, affecting 215 countries, areas, and territories worldwide [3]. COVID-19 is a highly contagious disease, which spreads from one human to another via droplets or direct contact. It has an incubation period ranging from 1 to 14 days. COVID-19 has been reported in all age groups. The majority of cases present with a flulike illness. Children are generally asymptomatic and present with milder disease compared to adults, with lower fatality rates [4-6]. Although the SARS-CoV-2 primarily causes acute respiratory illness with diffuse alveolar damage and interstitial pneumonia, it can affect multiple organs of the body such as the heart, liver, kidney, gastrointestinal tract, central nervous system, and blood [7]. The current pediatric data available on COVID-19-related acute kidney injury (AKI) are mostly anecdotal. The aim of this short review is to discuss what is currently known about the pediatric features of the renal disease and the different aspects of managing renal dysfunction in these patients especially in the context of limited resources and increased demand.

\section{Epidemiology of AKI in COVID-19}

Current information on kidney involvement in patients affected by COVID-19 is scarce. In a first initial report from China, the incidence of AKI in infected adults appeared to be around $0.5 \%$ and utilization of continuous renal replacement therapy (CRRT) was $0.8 \%$ [8]. Thereafter, an in-depth analysis on adult patients admitted to intensive care unit (ICU) reported an incidence of AKI of $5.1 \%$, with a concomitant prevalence of proteinuria and hematuria in COVID-19 patients. All reported parameters of renal damage correlated with survival [9]. This report did not describe the need for CRRT in this cohort. However, a recent study performed on 287 patients admitted to Hankou Hospital, Wuhan, China, showed a higher incidence of AKI (19.6\%) in COVID-19 patients and demonstrated a significant increase in mortality risk as compared to non-AKI COVID-19 subjects [10]. These rates of AKI are significantly lower than are reported in the general ICU population, which is around $40-50 \%$ [11]. This also demonstrates a highly significant correlation between survival and renal function.

Some reports on COVID-19 infection in children are already available from the Chinese epidemic. A recent review of 45 scientific papers showed that children have so far accounted for 1-5\% of diagnosed COVID-19 cases, with the minority being asymptomatic and the remaining $10 \%$ generally presenting with milder disease than adults and fatalities are rare [12]. The diagnostic findings, clinical course, and treatment of these patients have been similar to those of adults, even though only $0.6 \%$ of the pediatric cohort has been critically unwell with severe symptoms [13]. A high prevalence of severe disease (about $50 \%$ ) was seen in infants. The reduced susceptibility to severe disease in children has been attributed to less immune dysfunction and the immaturity of the angiotensin-converting enzyme 2 (ACE2) receptors, which are the binding sites of the SARS-CoV-2 $[14,15]$. Considering the evidence so far, it can be hypothesized that ventilation in children would be less aggressive and with less systemic involvement and renal dysfunction compared to adults. If the same proportion of AKI in COVID-19 adult cases is extrapolated to the pediatric age group, it would translate to an incidence of $2-3 \% \mathrm{AKI}$ in children with COVID-19. This is again less than the currently known epidemiology of $26 \%$ renal dysfunction in children admitted to pediatric intensive care unit (PICU) [16]. A recent report from a multicenter epidemiological survey of critically ill children (CAKE study) [17] found that AKI occurred in $18 \%$ of included patients even if little information was provided on classification and severity. It can also be proposed that children with comorbidities like congenital heart diseases and congenital renal diseases and renal transplant patients are at higher risk of renal complications with symptomatic COVID-19. Finally, children requiring aggressive fluid resuscitation are at risk of volume overload and can develop renal complications [18].

Recently, the National Health Service (NHS) in England has notified that a number of children with clinical features of toxic shock syndrome and atypical Kawasaki disease have been tested positive for COVID-19 [19]. This clinical picture represents a new phenomenon affecting previously asymptomatic children with SARSCoV-2 infection manifesting as a hyperinflammatory syndrome with multiorgan involvement similar to Kawasaki disease shock syndrome, mainly affecting the myocardium. A case series involving 8 such children has just been published in the Lancet[20]. A few of these children 
have required extracorporeal membrane oxygenation. AKI could develop as a part of the multisystem involvement probably secondary to the low cardiac output state, vasculitis, or both. AKI is also a known complication in Kawasaki disease and is reported in about one-third of these patients [21]. This syndrome has been named as paediatric inflammatory multisystem syndrome temporally associated with SARS-CoV-2 (PIMS-TS) in the UK and as multisystem inflammatory syndrome associated with COVID-19 (MIS-C) in the USA.

\section{Pathogenesis of AKI in COVID Patients}

The pathophysiology of kidney dysfunction in COVID-19 patients has been recently hypothesized to be secondary to cytokine storm, organ cross talk, systemic effects [22], and direct tubuloglomerular damage [23, 24]. Although generally speculative, evidence regarding these aspects is growing. The potential mechanisms of kidney injury in these patients can be discussed under 5 main categories:

1. Dehydration: Secondary to decreased fluid intake, vomiting, diarrhea, and overuse of diuretics to keep the lungs "dry." All predispose the patient to develop AKI.

2. Cytokine storm syndrome: In particular, high concentrations of interleukin- 6 have been described in COVID-19 patients, especially when lung disease has reached the criteria for acute respiratory disease syndrome (ARDS). In patients with cytokine storm, AKI can occur due to renal inflammation, increased vascular permeability, third space fluid loss, intra-abdominal hypertension, hypovolemia, and the subsequent shock.

3. Interaction between different organs: Aggressive ventilation of patients with severe hypoxemia is well known to be associated with altered kidney function as a component of organ cross talk [23]. Hemodynamic instability (in addition to ventilation issues may include cardiomyopathy or myocarditis) exacerbated by aggressive volume replacement, a positive fluid balance, and coinfection with bacterial pathogens with subsequent septic shock.

4. Systemic side effects of the virus: Recent initial reports have evidenced the possibility, confirmed by histopathological findings, that SARS-COV-2 virus may directly attack proximal tubules $[24,25]$.

5. Use of nephrotoxic drugs: Finally, nephrotoxicity induced by specific COVID-19 treatments (namely an-
Table 1. Monitoring of a pediatric patient with COVID-19 at risk for AKI

1 Assess volume status daily on clinical examination and non-invasive hemodynamic assessment by Doppler ultrasound or echocardiography

2 Individualize fluid balance targets in order to target children's optimal volume status if they present with volume depletion or avoid fluid overload in excess of $10 \%$. Fluid overload can lead to worsening of the patient's respiratory status

3 Measure serum urea, creatinine, and electrolytes at admission and then 24-48 hourly

\begin{tabular}{ll}
\hline 4 & Monitor urine output \\
\hline $5 \quad \begin{array}{l}\text { Review medications daily and withhold the ones that may } \\
\text { increase the risk of AKI. In particular, refer to NINJA } \\
\text { methodology and nephrotoxic list [28] }\end{array}$
\end{tabular}

COVID-19, novel coronavirus disease; AKI, acute kidney injury.

tiviral drugs and hydroxychloroquine) may be contributory. As in AKI of non-COVID etiology, close attention to the use of nephrotoxic drugs, monitoring drug levels, and reducing their use in high-risk patients is highly recommended especially in the setting of fluid overload and cumulative effects.

\section{Recognition, Monitoring, and Diagnosis of AKI in COVID-19 Patients}

It is imperative to predict, recognize, and manage AKI in COVID-19 patients at an early stage in order to prevent further parenchymal and glomerular involvement leading to irreversible cortical necrosis. AKI is detected in COVID-19 children by using the same KDIGO definition [26], as is used in non-COVID patients. AKI is regularly monitored by assessing urine output, fluid balance, renal functions (serum blood urea nitrogen and Creatinine), and electrolytes (Table 1). In COVID-19, children it is imperative to assess and optimize volume status to manage functional AKI, as they are at a high risk of severe dehydration during infectious diseases. At the same time, steps should be taken to prevent fluid overload, which can cause respiratory deterioration. Fluid replacement in children is particularly challenging and requires strict monitoring. Fluid resuscitation and ongoing hemodynamic management in these patients should follow the recently published Surviving Sepsis Campaign Interna- 
tional Guidelines for the Management of Septic Shock and Sepsis-Associated Organ Dysfunction in Children, which focuses on frequent assessment of fluid status [27].

\section{Treatment of AKI in COVID-19 Patients}

The management of AKI in children with COVID-19 is based on the same principles as AKI in non-COVID patients; however, it carries important practical considerations. Basic principles include fluid and hemodynamic assessment and review and modification of nephrotoxic medications as exampled by the NINJA project undertaken by Cincinnati Children's Hospital. Early institution of enteral nutrition, managing electrolyte, and acid-base abnormalities as per KDIGO guidelines and the surviving sepsis guidelines [26-28].

\section{Renal Replacement Therapy}

RRT in managing AKI in a pandemic state requires special attention to minimizing exposure of the healthcare staff to the virus while providing optimal patient care, using appropriate personal protective equipment (PPE) and disinfection of equipment. All staff should follow the hospital, local public health, and CDC recommendations for the use of PPE and safety guidelines during their interactions with patients.

\section{Basic Principle of "Protect Yourself and Your Team"}

Full PPE should always be worn when caring for COVID-19-positive or suspected patients during high disease prevalence. These recommendations have been changing rapidly and the staff involved in the care of these sick children undergoing RRT should receive regular updates on the recent recommendations and training in donning and doffing of PPE. The basic principle is to limit the exposure of the health-care professional to absolute minimum. This might require adjustments to the "usual" way of working including rationing of staffing members.

\section{Limiting Exposure to the Health-Care Staff}

In PICUs, where nurses and intensivists independently run the CRRT program, nephrologists and hemodialysis nurses do not need to have direct contact with patients, thereby limiting health-care staff exposure. However, in nephrology led CRRT programs, where the hemodialysis nurses set up and troubleshoot the machine, CRRT is set up outside the patient room. Then, the PICU nurse will take the machine into the room and connect the patient in order to minimize exposure and use of limited PPEs. Use of telemedicine can be valuable, if available, to help the dialysis nurse and nephrologist to troubleshoot remotely rather than coming in direct contact with the patient.

\section{Disinfection of the RRT Equipment}

Some hospitals make a local decision to change filters more than the recommended $72 \mathrm{~h}$. All dialysis equipment should be cleaned with the disinfectant as per the manufacturer/local hospital use/CDC recommendations before the equipment is moved out of the isolation room. All disposable RRT equipment and consumables should be carefully discarded as per the local hospital policy or CDC recommendations.

\section{When to Initiate?}

Children at presentation can be severely volume depleted owing to fever, reduced fluid intake before hospitalization, and then fluid restriction and diuretic therapy after hospital admission. Sodium can increase significantly ranging from 155 to $160 \mathrm{meq} / \mathrm{L}$. Hence, it is important to fill the tank and make the patients euvolemic before considering RRT. Once the volume status is optimized, indications for commencement of RRT are the same as for non-COVID patients - electrolyte imbalance, azotemia, fluid overload, and acid-base disturbances not responding to the medical management. Another important consideration while deciding to initiate RRT in COVID-19 children is the increased exposure to the healthcare professionals and need for additional staff and machinery. In a recent systematic review and individual patient data meta-analysis of randomized clinical trials, Gaudry et al. [29] concluded that the timing of RRT initiation does not affect survival in critically ill patients with severe AKI in the absence of urgent indications for RRT. Therefore, the time to initiate RRT should be based on the risk-benefit ratio, which is particularly challenging in critically ill children. However, a practical factor currently determining the decision to initiate RRT in COVID-19 children is the availability of resources, which might get depleted very quickly, especially in pediatric centers that have started admitting adult patients with COVID-19, owing to the overwhelming demand for both the machines and consumables. Hence, delaying the initiation of RRT, while closely monitoring the patient with AKI, can reduce the need for RRT and associated health resources. 
Which Modality Is Preferable for Treatment of AKI COVID-19 Pandemic?

The decision to choose between intermittent and continuous RRT depends, as usual, on the hemodynamic status of the patient and available resources. In theory, convection-based clearance appears to be more effective in removing the cytokines, which are proposed to be one of the reasons for the severity of illness in COVID-19 patients, as compared to diffusion-based clearance. However, there are not enough data so far to support this theory [30]. Moreover, convection being non-selective, it may contribute to removing both pro-inflammatory cytokines and anti-inflammatory ones. No difference in outcomes has been shown among the various modalities of CRRT (continuous venovenous hemofiltration $[\mathrm{CVVH}]$ or continuous venovenous hemodialysis [CVVHD] or continuous venovenous hemodiafiltration [CVVHDF] ) in adult patients with AKI [31]. Similarly, in children, the modality of choice appears to be center dependent without significant clinical differences, and in the only available report in the pediatric population, $21 \%$ of patients received CVVH, $48 \%$ received CVVHD, and $30 \%$ received CVVHDF [32]. It is possible that when blood flow rate is limited due to small vascular access in children, a diffusive technique may help to optimize the filter life span $[33,34]$. Similarly, no additional benefit has been reported with hybrid therapies such as prolonged intermittent renal replacement therapy (PIRRT), sustained low efficiency dialysis (SLED), and sustained low efficiency diafiltration (SLED-f) [35]. Hence, it is recommended to continue using the same modality which the institution is comfortable with [36]. Any last-minute changes in the existing CRRT guidelines during the COVID-19 pandemic might create more chaos and confusion. Also, training of medical staff with any new modality at this time is not recommended. It can increase the chance of medical errors and compromise the treatment of critically sick patients with COVID-19.

What Are the Practical Points in the RRT Provision during COVID-19 Pandemic?

The advantages of CRRT include better tolerance in hemodynamic unstable children and accuracy in fluid removal. CRRT depending on expertise, staffing, and resources would remain the preferred dialysis modality among critically ill patients with AKI in this pandemic. This is true from the logistics point of view as not every ICU has facilities to deliver intermittent hemodialysis (IHD) (reverse osmosis - RO system). IHD requires 1:1 nursing, use of PPE for each nurse, and constant expo- sure, while 1 nurse can manage more than 1 patient on CRRT, thereby limiting the use of PPE and exposure to the frontline nursing staff. Therefore, even among patients who are hemodynamically stable and could tolerate IHD, CRRT or PIRRT may be the preferred modality. Patients can be initiated on CRRT with machines at the bedside in the isolation room as per standard conditions, or, using extended tubing, CRRT machines can be placed outside the room with the attended risks of more frequent clotting, decreased sensitivity of pressure alarms, and inadvertent disconnections. The latter setup is not particularly feasible in children due to the priming volume of the machine that risks becoming excessively large. However, the management of CRRT machines from outside the isolation room might reduce the frequent exposure of healthcare personnel and thereby reduces the use of PPE. However, in the condition where the hospital is overwhelmed with COVID-19 patients, there is a huge possibility of exhaustion of CRRT machines, infusion pumps, consumables (circuit, hemofilter, dialysate, replacement solutions, and anticoagulants), and unavailability of staff with adequate expertise to run CRRT. Though the pediatric numbers are low, the shortage of CRRT machines, consumables, and trained staff is even more pronounced for pediatric patients as most of the devices and staff are shared or diverted to adult ICUs. When the supply of CRRT machines and consumables outstrips the demand, the use of IHD in the selected group of hemodynamically stable patients with $\mathrm{AKI} /$ fluid overload can be a feasible option.

\section{Special Considerations in Prescribing CRRT in}

\section{Resource-Limited Setting}

Not uncommonly, during the course of this prolonged pandemic, provision for CRRT has been limited due to over-utilization of resources (i.e., machines, monitors, filters, circuits, infusion pumps, replacement or dialysis fluid, and anticoagulant).

When CRRT Machines Are Limited

1. Prolonged intermittent treatments using the conventional CRRT machines can be delivered (e.g., 8-10 h rather than continuous) with higher than usual exchange rates - 50-60 mL/kg/h - instead of the recommended dose of $35-45 \mathrm{~mL} / \mathrm{kg} / \mathrm{h}$ or $2 \mathrm{~L} / 1.73 \mathrm{~m}^{2} / \mathrm{h}$, which is conceived to be delivered in an ideal 24 -h session $[37,38]$. In resource-limited scenario, this might be practically very useful to distribute available resources applying the same machine to $2-3$ patients in a day. 
2. Machines can be rotated between patients every $24 \mathrm{~h}$ or whenever the circuit clots to minimize use of filters and circuits.

3. Consider a "filter holiday" if spontaneous urine output is $>0.5 \mathrm{~mL} / \mathrm{kg} / \mathrm{h}$ and fluid status and potassium levels are controlled.

When CRRT Consumables Are Limited

1 . When there is limited supply of replacement or dialysis fluid, lower exchange rates $(20 \mathrm{~mL} / \mathrm{kg} / \mathrm{h})$ can be used especially in AKI patients who require CRRT for fluid overload and are not hypermetabolic.

2. In specific circumstances, fluids from one company can be used with the CRRT machine from another commercial company. In our institution, we used replacement fluid from Aquarius machine (Aquasol) with the CRRT machine from Baxter (Prismaflex). When there is very limited supply of any kind of replacement fluid, certain institutes may have the inherent capability of developing their own fluid by mixing saline and dextrose with various concentrations of potassium, sodium bicarbonate, magnesium chloride, calcium chloride, and phosphate. One needs to ensure the sterility and stability of the fluid prepared. The biggest challenge will be the amount of electrolytes required to prepare a bag of fluid; in challenging pandemic scenarios, these electrolytes might not be available in the pharmacy in adequate quantity to prepare a large number of bags of replacement/dialysis fluid.

Hence, the most important factor that determines the provision of RRT in the setting of COVID outbreak is the availability of resources. These are unprecedented circumstances and we need to adapt to using our resources with the utmost efficacy in innovate ways to provide RRT with limited machines, consumables, and trained staff. The RRT prescription in terms of venous access, blood flow rate, and CRRT dose in general PICU setting as well as in the case of COVID-19 are described in Table 2.

Anticoagulation in CRRT - Do the Filters Clot More Frequently in Patients on CRRT with COVID Disease?

The importance of anticoagulation in CRRT circuits was shown by Brophy et al. [39] in the data from the Prospective Pediatric CRRT (ppCRRT) Registry, which reinforced the fact that CRRT circuits clot more frequently if no anticoagulation is used. COVID-19 has been reported to be a hypercoagulable state (increased d-dimer concentration) in the adult population [40]. Hence, the guidelines support the use of thromboprophylaxis in these pa- tients to prevent thrombotic complications like pulmonary embolism and venous thromboembolism [41]. In addition, the very high inflammatory response in these patients due to cytokine storm also contributes to repeated filter clotting. An anecdotal problem in the delivery of CRRT to patients with COVID-19 has been frequent filter clotting, leading to consumption of already restrained resources, increased downtimes, and decreased efficacy of treatment. The following anticoagulants and their combinations can be used to prevent filter clotting.

1. Unfractionated heparin (UFH) - Heparin remains the most commonly used anticoagulant in these patients as a number of these patients develop pulmonary emboli or deep vein thrombosis and are started on systemic infusion of UFH. If patients are not on a systemic heparin infusion, a pre-filter bolus of UFH (20 units per kg), followed by an infusion of heparin at the dose of 20-30 units per kg per hour (higher than the usual dose of 10-20 units per kg per h) should be started. Aim for activated clotting time (ACT) of 180-220 $s$, if activated clotting time is low and the filter clots, increasing the dose of UFH by $10-20 \%$ of the previous dose is recommended. Side effects related to UFH need to be borne in mind especially increased risk of bleeding, heparin resistance, and heparin-induced thrombocytopenia.

2. Combination of heparin and prostacyclin - In order to minimize the dose of heparin used for anticoagulation as well as heparin-induced side effects while addressing the issue of increased filter clotting, a combination of heparin and prostacyclin can be used (both are administered prefilter). Prostacyclin helps to reduce filter clotting by its anti-platelet and is heparin sparing [42]. In this setting, heparin at 10 units per kg per hour is combined with prostacyclin given at the rate of $4 \mathrm{ng} /$ $\mathrm{kg} / \mathrm{min}$.

3. Regional citrate anticoagulation (RCA) - Citrate is another anticoagulant that prolongs the circuit life and reduces the hemorrhagic complications of heparin [43-45]. If repeated clotting is observed with a standard RCA protocol, lower ionized calcium levels $(0.2$ $\mathrm{mmol} / \mathrm{L}$ ) in the CRRT circuit can be targeted (the usual target is $0.3-0.4 \mathrm{mmol} / \mathrm{L}$ ). A higher concentration of citrate, $4 \%$ instead of $0.5 \%$, can be used in order to reduce convection and transmembrane pressure. Since many adult patients with COVID-19 have deranged liver function tests, citrate accumulation can occur in these patients leading to severe hypocalcemia or citrate lock. Therefore, strict monitoring of calcium is required while using citrate as an anticoagulant. 
Table 2. Special considerations in patients with COVID-19 while using various RRT modalities

Staffing, PPE, and disinfection

Full PPE to be worn while managing COVID patients

Minimum exposure to health-care personnel

Disinfection of all dialysis equipment should be done as per hospital guidelines or CDC recommendations. Meticulously discard all disposable RRT equipment and consumables as per hospital policy

General PICU setting

COVID-19 setting

CRRT prescription

Vascular access size and

site

Size of vascath depends on the weight of the child

Right IJV is the preferred site of insertion due to ease of insertion with ultrasound guidance and relatively bigger sized catheters can be inserted, lesser chances of kinking, which ensure better flows and prevents circuit clotting

Blood flow rate (BFR) Prescribed according to the weight of the child neonates $-8-12 \mathrm{~mL} / \mathrm{kg} / \mathrm{min}$, children $-4-8 \mathrm{~mL} / \mathrm{kg} / \mathrm{min}$, older children $-2-4 \mathrm{~mL} / \mathrm{kg} / \mathrm{min}$

CRRT dose $\quad 35-45 \mathrm{~mL} / \mathrm{kg} / \mathrm{h}$ or $2 \mathrm{~L} / 1.73 \mathrm{~m}^{2} / \mathrm{h}$
Size of vascath depends on the weight of the child

Right IJV is preferred. Along with other benefits, it is easier to maintain and access in case of COVID patients

Prescribed according to weight of the child. Blood flow might have to be increased in order to counteract frequent filter clotting

In resource-limited settings:

1. When machines are limited, 1 CRRT machine can used to provide RRT to multiple patients for restricted hours per day (8-12 h), with increased dose of $50-60 \mathrm{~mL} / \mathrm{kg} / \mathrm{h}$ or $4-5 \mathrm{~L} / 1.73$ $\mathrm{m}^{2} / \mathrm{h}$

In severely ill patients where there is a very high metabolic rate, dose may be increased to $50-60 \mathrm{~mL} / \mathrm{kg} / \mathrm{h}$ or $4-5 \mathrm{~L} / 1.73 \mathrm{~m}^{2} / \mathrm{h}$

2. If consumables are limited, low-dose CRRT $20-30 \mathrm{~mL} / \mathrm{kg} / \mathrm{h}$ should be given for longer period of time $(24 \mathrm{~h})$. We can also make our own dialysate fluid

According to center's preference and expertise - CVVH or CVVHD or CVVHDF
Use the same modality which the center is comfortable with

If CVVH is practiced, higher pre-dilution dose can be used to counteract filter clotting

If CVVHDF is practiced, lower doses of post-dilution can be used to minimize bubble trap clotting

In resource-limited settings, IHD or PIRRT can be used

Filters clot more frequently - higher doses of anticoagulants/ combination of anticoagulants might have to be used

$30-50 \mathrm{~mL} / \mathrm{kg}$ or $1,200 \mathrm{~mL} / \mathrm{m}^{2}$ to achieve better solute clearance in hypercatabolic state of COVID-19

PD prescription

PD dose $\quad 10-20 \mathrm{~mL} / \mathrm{kg}$ or $300-600 \mathrm{~mL} / \mathrm{m}^{2}$

Increased or better solute clearance. Depends on condition of oxygenation and fluid overload

Concentration of glucose $\quad 2.5 \%$

in PD fluid

Higher concentration $2.5-4.25 \%$ to achieve better solute clearance

COVID-19, novel coronavirus disease; CRRT, continuous renal replacement therapy; CVVH, continuous venovenous hemofiltration; CVVHD, continuous venovenous hemodialysis; CVVHDF, continuous venovenous hemodiafiltration; IHD, intermittent hemodialysis; IJV, internal jugular vein; PD, peritoneal dialysis; PICU, pediatric intensive care unit; PIRRT, prolonged intermittent renal replacement therapy; PPE, personal protective equipment; RRT, renal replacement therapy.

4. Combination of RCA and systemic unfractionated heparin - In the event of frequent filter clotting, despite optimal doses of heparin or citrate used independently, experienced centers can try a combination of
RCA (in the usual recommended dose as for non-COVID patient) alongside systemic unfractionated heparin infusion. 
5. Low-molecular-weight heparin (LMWH) - There might be instances, where the availability of infusion pumps to deliver heparin infusion might be inadequate. In these circumstances, LMWH (dalteparin and enoxaparin) administered subcutaneously in the treatment dose might be an option. If filters still clot on LMWH, RCA might be added to optimize filter halflife. Some centers administer enoxaparin intravenously in children in order to reduce the discomfort of subcutaneous administration. The usual measures to reduce filter clotting in non-COVID patients will apply to children with COVID-19 as well:

1. Select larger catheters and address all the catheter-related problems like location, leakage, bending, and kinking.

2. Set higher blood flow rates.

3. Use pre-dilution replacement fluid during CVVH to reduce filtration fraction and clotting.

4. Preferential use of filters with a larger surface area aiming to reduce transmembrane pressure or in smaller children when smaller circuits are applied use diffusive modalities.

5. While using CVVHDF, apply some post-filter reinfusion in order to prevent clotting in the bubble trap.

\section{Can Peritoneal Dialysis Be Used if Supplies of CRRT}

Run Low?

Peritoneal dialysis (PD) appears to be a feasible alternative to CRRT in children with AKI, especially when limitation of resources is an issue and anticoagulation of extracorporeal circuits is challenging. There is sufficient evidence to prove that $\mathrm{PD}$ is equally effective as other forms of renal replacement therapies [46], and in children, there is a lot of experience with this technique in different settings $[47,48]$. PD requires relatively less equipment, consumables, and resources as compared to other forms of RRT. Staff can be easily trained to perform PD. It can be run manually or by using an automated dialyzer after inserting Tenckhoff/Seldinger technique PD catheter. In COVID-19 patients, automated PD with a cycler should be preferred if available, as it minimizes the contact between health-care worker and the patient. The recommended dose of PD is 10-20 mL/kg (300-600 $\mathrm{mL} / \mathrm{m}^{2}$ ), but in case of high catabolic states, aggressive PD regimen $\left(30-50 \mathrm{~mL} / \mathrm{kg}\right.$ or $\left.1,200 \mathrm{~mL} / \mathrm{m}^{2}\right)$ can be used in order to ensure adequate solute removal. PD inflow time can vary from 5 to $10 \mathrm{~min}$, followed by dwell time of 30 60 min and PD outflow time of 20-25 min. Dwell time can also be increased in order to target better solute clearance for adequate metabolic control while keeping in mind that prolonged dwell time can result in fluid retention. Also, after a few exchanges, the dextrose concentration in the PD fluid can be increased from 2.5 to $4.25 \%$ to achieve more efficient ultrafiltration. Heparin 250-1,000 units per liter of dialysate can be added as an anticoagulant to the PD fluid, and potassium chloride 3-4 mmol/L can be added to PD fluid in case of hypokalemia. Problems associated with PD catheters in critically sick children are pericatheter leaks, peritonitis, blockage of catheter, and unpredictable fluid removal [49]. In addition, PD can interfere with respiratory mechanics and can compromise ventilation due to raised intra-abdominal pressures due to fluid retention. To overcome this issue, some units routinely measure intra-abdominal pressure to decide about how long and how much to drain the fluid. Smaller PD volumes with shorter dwell times can solve this problem. Moreover, performing PD can be problematic in prone ventilation, which is routinely followed in many ARDS patients with moderate to severe hypoxemia. The catheter is placed with the patient in the supine position. While carrying out PD in the prone position, one needs to keep a close eye on associated risks including kinking and leakage of the catheter, potentially leading to skin breakdown and pressure sores. The solute clearance and the precision of fluid removal may not be as good as CRRT, but close monitoring of fluid balance and careful drug administration (i.e., antibiotics, amino acids with nutrition, and sedatives) may help to solve this issue.

\section{Other Extracorporeal Therapies}

One of the postulated concerns associated with $\mathrm{CO}$ VID-19 is the cytokine storm resulting in systemic inflammatory response syndrome and capillary leak syndrome resulting in organ damage, such as ARDS, liver injury, cardiac injury, and AKI. Extracorporeal therapies such as hemoperfusion and hemadsorption can theoretically be beneficial in COVID-19 patients with AKI as they remove the cytokines and other inflammatory mediators from the blood, offering hemodynamic and organ support [22]. However, there is very little evidence to support that immunomodulation through removal of cytokines will help in outcomes.

CytoSorb has been recommended by the Italy Brescia Renal COVID Task Force in patients with Stage 3 AKI receiving CRRT [50] as well as the Chinese Clinical Guidance for COVID-19 Pneumonia Diagnosis and Treatment [51]. WHO has introduced CytoSorb in the list of 
experimental treatments in COVID-19 patients [52]. Only few reports on critically ill (non-COVID-19) children have described this therapy as safe and potentially effective [53-55]. In the opinion of the authors, at the moment, this therapy would require further evaluation in COVID-19 children. Outside a research protocol or rescue/compassionate application, it should not be considered. Furthermore, this therapy is not widely available, it has not been approved in certain countries and it cannot be considered in routine care.

\section{Ethical Considerations}

Fortunately, most children are reported to recover well from this illness. Hence, the triaging decision-making required of adult colleagues in the COVID-19 pandemic has not been necessary in any pediatric intensive care setup. However, the diversion of all equipment/consumables and redeployment of staff to cater for the vast number of adult patients may lead to a deficit of resources for children. If all RRT equipment and consumables are occupied, at a point when a child requires RRT, the decision to withhold the life-sustaining treatment in a child might be very difficult. Similarly, reverse triage to withhold RRT from 1 patient to maximize opportunity to the other requires similar oversight, discussion, and ethical support. It should also be considered that since it is affecting countries with ethnically diverse populations, a consistent risk is present that resource utilization is disproportionate in minority groups [56]. Decision-making during these difficult circumstances must follow the ethical principles set out for any pandemic. They need to be open, transparent, inclusive, and accountable. Support of institution's ethical committee is strongly recommended.

\section{Conclusion}

In summary, incidence of AKI in children affected by SARS-CoV-2 is unknown, and it is probably lower than in adults. However, in those cases presenting with the same severity of illness as adults, the same clinical picture of renal involvement may be expected. Initial reports of AKI in COVID-19 patients are already alerting that the mortality is significantly increased when the renal dysfunction is severe. Early diagnosis and timely treatment of potential triggers of renal injury (e.g., dehydration, fluid overload, and drug toxicity) are crucial and currently largely unexplored in COVID-19 children. In established anuric pediatric AKI, RRT remains the mainstay of renal support. Specific aspects to be considered in COVID-19 children requiring dialysis include the utilization of $\mathrm{PD}$, the shortage of resources due to overutilization in adult centers, the occurrence of particularly frequent filter clotting, and need for addressing specific anticoagulation strategies. Finally, although pragmatic utilization of available resources and safety of health-care workers is important in these challenging circumstances, particular attention should be posed not to avoid or delay RRT in a COVID-19 child with severe AKI. The delay to provide this kind of treatment in severely ill children may cause an unacceptable increase in the mortality of such frail patients. These are unprecedented times, the spectrum of clinical presentation of children affected by COVID-19 is evolving, and we, as clinicians, will need to adapt to this new unknown.

\section{Statement of Ethics}

The paper is exempt from ethical committee approval as it is a review article and does not involve data on any patients.

\section{Conflict of Interest Statement}

Z.R. and A.D. are review board members and Associate Editor for this journal (Blood Purification). The authors have no other conflicts of interest to declare.

\section{Funding Sources}

The authors did not receive any funding.

\section{Author Contributions}

A.D.: Concept and design, literature review, and prepared the manuscript. M.B.: Prepared the manuscript and edited the final version. Z.R.: Edited the manuscript and final approval of the version to submitted.

\footnotetext{
References $\quad 1$ Zhu N, Zhang D, Wang W, Li X, Yang B, Song $\mathrm{J}$, et al. A novel coronavirus from patients with pneumonia in China, 2019. N Engl J Med. 2020;382(8):727-33.

2 Li Q, Guan X, Wu P, Wang X, Zhou L, Tong $\mathrm{Y}$, et al. Early transmission dynamics in $\mathrm{Wu}-$ han, China, of novel coronavirus-infected pneumonia. N Engl J Med. 2020;382(13): 1199-207.
} 
3 World Health Organisation. Coronavirus disease (COVID-19) outbreak situation. 2020 [cited 2020 May 3]. Available from https:// www.who.int/emergencies/diseases/novelcoronavirus-2019.

4 Dong Y, Mo X, Hu Y, Qi X, Jiang F, Jiang Z, et al. Epidemiological characteristics of 2143 pediatric patients with 2019 coronavirus disease in China. Pediatr. Forthcoming 2020.

5 Zimmerman P, Curtis N. Coronavirus infections in children including COVID-19. Pediatr Infect Dis J. 2020;39(5):355-68.

6 Liu W, Zhang Q, Chen J, Xiang R, Song H, Shu S, et al. Detection of COVID-19 in children in early January 2020 in Wuhan, China. N Engl J Med. 2020;382(14):1370-1.

7 Wang D, Hu B, Hu C, Zhu F, Liu X, Zhang J, et al. Clinical characteristics of 138 hospitalized patients with 2019 novel coronavirus-infected pneumonia in Wuhan, China. JAMA. 2020;323(11):1061-9.

8 Guan WJ, Ni ZY, Hu Y, Liang WH, Ou CQ, $\mathrm{He} J \mathrm{X}$, et al. China medical treatment expert group for COVID-19. Clinical characteristics of coronavirus disease 2019 in China. N Engl J Med. Forthcoming 2020 Feb 28.

9 Cheng Y, Luo R, Wang K, Zhang M, Wang Z, Dong $\mathrm{L}$, et al. Kidney disease is associated with in-hospital death of patients with COVID-19. Kidney Int. 2020 Mar 20;97(5):829-38.

10 Xiao G, Hu H, Wu F, Sha T, Huang Q, Han J, et al. Acute kidney injury in patients hospitalized with COVID-19 in Wuhan, China: a single-center retrospective observational study. medRxiv. 2020.

11 Hoste EA, Bagshaw SM, Bellomo R, Cely CM, Colman R, Cruz DN, et al. Epidemiology of acute kidney injury in critically ill patients: the multinational AKI-EPI study. Intensive Care Med. 2015 Aug;41(8):1411-23.

12 Ludvigsson JF. Systematic review of COVID-19 in children shows milder cases and a better prognosis than adults. Acta Paediatr. 2020 Jun;109(6):1088-95.

13 Dong Y, Mo X, Hu Y, Qi X, Jiang F, Jiang Z, et al. Epidemiology of COVID-19 among children in China. Pediatrics. 2020;145(6): e20200702.

14 Lu X, Zhang L, Du H, Zhang J, Li YY, Qu J, et al. SARS-CoV-2 infection in children. N Engl J Med. 2020;382(17):1663-5.

15 Xu Y, Li X, Zhu B, Liang H, Fang C, Gong Y, et al. Characteristics of pediatric SARS-CoV-2 infection and potential evidence for persistent fecal viral shedding. Nat Med. 2020;26(4): 502-5.

16 Kaddourah A, Basu RK, Bagshaw SM, Goldstein SL; AWARE Investigators. Epidemiology of acute kidney injury in critically Ill children and young adults. N Engl J Med. 2017 Jan 5;376(1):11-20.

17 González-Dambrauskas S, Vásquez-Hoyos P, Camporesi A, Díaz-Rubio F, Piñeres-Olave $\mathrm{BE}$, Fernández-Sarmiento $\mathrm{J}$, et al. Critical coronavirus and kids epidemiology (CAKE) study. Pediatric critical care and COVID19. Pediatrics. 2020 Jun:e20201766.
18 Alobaidi R, Morgan C, Basu RK, Stenson E, Featherstone R, Majumdar SR, et al. Associations between fluid balance and outcomes in critically Ill children: a protocol for a systematic review and meta-analysis. Can J Kidney Health Dis. 2018 Mar 1;4(3): 2054358117692560.

19 PICS Statement. Increased number of reported cases of novel presentation of multisystem inflammatory disease. 2020 [cited 2020 May 3]. Available from: https://picsociety.uk/wp-content/uploads/2020/04/ PICS-statement-re-novel-KD-C19-presentation-v2-27042020.pdf.

20 Riphagen S, Gomez X, Gonzalez-Martinez C, Wilkinson N, Theocharis P. Hyperinflammatory shock in children during COVID-19 pandemic. Lancet. 2020;395(10237):1607-8.

21 Chuang GT, Tsai IJ, Lin MT, Chang LY. Acute kidney injury in patients with Kawasaki disease. Pediatr Res. 2016;80(2):224-7.

22 Ronco C, Reis T. Kidney involvement in COVID-19 and rationale for extracorporeal therapies. Nat Rev Nephrol. 2020;16(6):308-10.

23 Joannidis M, Forni LG, Klein SJ, Honore PM, Kashani K, Ostermann M, et al. Lung-kidney interactions in critically ill patients: consensus report of the acute disease quality initiative (ADQI) 21 workgroup. Intensive Care Med. 2020 Apr;46(4):654-72.

24 Su H, Yang M, Wan C, Yi L, Tang F, Zhu H, et al. Renal histopathological analysis of 26 postmortem findings of patients with COVID-19 in China. Kidney Int. 2020;98(1): 219-27.

25 Pan XW, Xu D, Zhang H, Zhou W, Wang LH, Cui XG. Identification of a potential mechanism of acute kidney injury during the $\mathrm{CO}$ VID-19 outbreak: a study based on single-cell transcriptome analysis. Intensive Care Med. 2020;46(6):1114-6.

26 Kellum JA, Lameire N, Aspelin P, Barsoum RS, Burdmann EA, Goldstein SL, et al. Kidney disease: Improving global outcomes (KDIGO) acute kidney injury work group. Kidney Int Suppl. 2012;2:1-138.

27 Weiss SL, Peters MJ, Alhazzani W, Agus MSD, Flori HR, Inwald DP, et al. Surviving Sepsis campaign international guidelines for the management of septic shock and sepsisassociated organ dysfunction in children. Pediatr Crit Care Med. 2020;21(2):e52-106.

28 Goldstein SL, Mottes T, Simpson K, Barclay C, Muething S, Haslam DB, et al. A sustained quality improvement program reduces nephrotoxic medication-associated acute kidney injury. Kidney Int. 2016;90(1):212-21.

29 Gaudry S, Hajage D, Benichou N, Chaïbi K, Barbar S, Zarbock A, et al. Delayed versus early initiation of renal replacement therapy for severe acute kidney injury: a systematic review and individual patient data meta-analysis of randomised clinical trials. Lancet. 2020; 395(10235):1506-15.

30 Honore PM, Hoste E, Molnár Z, Jacobs R, Joannes-Boyau O, Malbrain MLNG, et al. Cytokine removal in human septic shock: where are we and where are we going? Ann Intensive Care. 2019;9(1):56.

31 Friedrich JO, Wald R, Bagshaw SM, Burns KE, Adhikari NK. Hemofiltration compared to hemodialysis for acute kidney injury: systematic review and meta-analysis. Crit Care. 2012 Aug 6;16(4):R146.

32 Symons JM, Chua AN, Somers MJ, Baum MA, Bunchman TE, Benfield MR, et al. Demographic characteristics of pediatric continuous renal replacement therapy: a report of the prospective pediatric continuous renal replacement therapy registry. Clin J Am Soc Nephrol. 2007;2(4):732-8.

33 Hackbarth R, Bunchman TE, Chua AN, Somers MJ, Baum M, Symons JM, et al. The effect of vascular access location and size on circuit survival in pediatric continuous renal replacement therapy: a report from the PPCRRT registry. Int J Artif Organs. 2007; 30(12):1116-21.

34 Lorenzin A, Garzotto F, Alghisi A, Neri M, Galeano D, Aresu S, et al. CVVHD treatment with CARPEDIEM: small solute clearance at different blood and dialysate flows with three different surface area filter configurations. Pediatr Nephrol. 2016 Oct;31(10):1659-65.

35 Baldwin I, Naka T, Koch B, Fealy N, Bellomo R. A pilot randomised controlled comparison of continuous veno-venous haemofiltration and extended daily dialysis with filtration: effect on small solutes and acid-base balance. Intensive Care Med. 2007;33(5):830-5.

36 American Society of Nephrology. Recommendations on the care of hospitalised patients with COVID-19 and kidney failure requiring renal replacement therapy. 2020 [cited 2020 April 13]. Available from: https:// www.asn-online.org/covid-19/ASN\#ASN_ Recommendations.

37 Palevsky PM, O'Connor TZ, Chertow GM, Crowley ST, Zhang JH, Kellum JA, et al. Intensity of renal replacement therapy in acute kidney injury: perspective from within the acute renal failure trial network study. Crit Care. 2009;13(4):310.

38 RENAL Replacement Therapy Study Investigators; Bellomo R, Bellomo R, Cass A, Cole L, Finfer S, Gallagher M, et al. Intensity of continuous renal-replacement therapy in critically ill patients. N Engl J Med. 2009;361(17): 1627-38

39 Brophy PD, Somers MJ, Baum MA, Symons JM, McAfee N, Fortenberry JD, et al. Multicentre evaluation of anticoagulation in patients receiving continuous renal replacement therapy (CRRT). Nephrol Dial Transplant. 2005;20(7):1416-21.

40 Tang N, Li D, Wang X, Sun Z. Abnormal coagulation parameters are associated with poor prognosis in patients with novel coronavirus pneumonia. J Thromb Haemost. 2020;18(4): 844-7.

41 Spyropoulos AC, Ageno W, Barnathan ES. Hospital-based use of thromboprophylaxis in patients with COVID-19. Lancet. 2020; 395(10234):e75. 
42 Deep A, Zoha M, Dutta Kukreja P. Prostacyclin as an anticoagulant for continuous renal replacement therapy in children. Blood Purif. 2017;43(4):279-89.

43 Fernández SN, Santiago MJ, López-Herce J, García M, Del Castillo J, Alcaraz AJ, et al. Citrate anticoagulation for CRRT in children: comparison with heparin. Biomed Res Int. 2014;2014:786301.

44 Sik G, Demirbuga A, Annayev A, Citak A. Regional citrate versus systemic heparin anticoagulation for continuous renal replacement therapy in critically ill children. Int J Artif Organs. 2020;43(4):234-41.

45 Liet JM, Allain-Launay E, Gaillard-LeRoux B, Barrière F, Chenouard A, Dejode JM, et al. Regional citrate anticoagulation for pediatric CRRT using integrated citrate software and physiological sodium concentration solutions. Pediatr Nephrol. 2014;29(9):1625-31.

46 Chionh CY, Soni SS, Finkelstein FO, Ronco C Cruz DN. Use of peritoneal dialysis in AKI: a systematic review. Clin J Am Soc Nephrol. 2013;8(10):1649-60.

47 Mishra OP, Gupta AK, Pooniya V, Prasad R, Tiwary NK, Schaefer F. Peritoneal dialysis in children with acute kidney injury: a developing country experience. Perit Dial Int. 2012; 32(4):431-6.

48 Li H, Yang S, Jin L, Wang Z, Xie L, Lv J, et al. Peritoneal dialysis treatment in small children with acute kidney injury: experience in Northwest China. Blood Purif. 2019;48(4): 315-20.

49 Ponce D, Caramori JT, Barretti P, Balbi AL. Peritoneal dialysis in acute kidney injury: Brazilian experience. Perit Dial Int. 2012;32(3): 242-6.

50 Brescia Renal Covid Task Force; Alberici F, Delbarba E, Manenti C, Econimo L, Valerio F, et al. Management of patients on dialysis and with kidney transplant during COVID-19 infection. 2020 [cited 2020 April 20]. Available from: https://www.era-edta.org/en/wp-content/uploads/2020/03/COVID_guidelines_ finale_eng-GB.pdf.

51 China National Health Commission. Chinese clinical guidance for COVID-19 pneumonia diagnosis and treatment (7th ed.). 2020 [cited 2020 April 25]. Available from: http://kjfy. meetingchina.org/msite/news/show/ $\mathrm{cn} / 3337 . \mathrm{html}$.
52 WHO. R\&D blueprint COVID 19 experimental treatments. 2020 [cited 2020 April 30]. Available from: https://www.who.int/docs/ default-source/coronaviruse/covid-classification-of-treatment-types-rev.pdf.

53 Bottari G, Merli P, Guzzo I, Stoppa F, Ruggeri A, Di Nardo M, et al. Multimodal therapeutic approach of cytokine release syndrome developing in a child given chimeric antigen receptor-modified T cell infusion. Crit Care Explor. 2020 Jan 29;2(1):e0071.

54 Saparov A, Sazonov V, Tobylbaeva Z, Isakov S, Bekpan A, Autalipov D, et al. First successful hemoadsorption using CytoSorb ${ }^{\circledR}$ in a septic pediatric patient in Kazakhstan: a case report. Int J Artif Organs. 2019 Jun;42(6): 315-7.

55 Bottari G, Guzzo I, Marano M, Stoppa F, Ravà L, Di Nardo M, et al. Hemoperfusion with Cytosorb in pediatric patients with septic shock: a retrospective observational study. Int J Artif Organs. 2020 Jan 31:039139882090246.

56 Harman K, Verma A, Cook J, Radia T, Zuckerman M, Deep A, et al. Ethnicity and COVID-19 in children with comorbidities. Lancet Child Adolesc Health. 2020;4(7):e24-5. 